\title{
The Density of Gaps in the Seal Joint in Elastic Contact of Microasperities
}

\author{
Peter M. Ogar ${ }^{\mathrm{a}}$, Denis B. Gorokhov ${ }^{\mathrm{b}}$, Artem S. Kozhevnikov ${ }^{\mathrm{c}}$ \\ Bratsk State University, Bratsk, Russia \\ aogar@brstu.ru, bdenis_gorohov@mail.ru, ${ }^{\mathrm{c}}$ kozhevnikovart@inbox.ru
}

\begin{abstract}
Initially, a contact between a rigid rough surface and a homogeneous elastic half-space without taking into account the mutual influence of microasperities is considered. To determine the dependence of the density of gaps in the joint on dimensionless the force elastic geometrical parameter, the discrete roughness model presented by microasperities in the form of equal spherical segments with the height distribution corresponding to the bearing profile curve of the real surface is used. To determine the volume of intercontact space, the volumes of gaps attributable to the single contacting or noncontacting asperities are determined. In this case the equations describing the sections of surfaces of contacting or noncontacting asperities and the half-space under load are used. Then we consider a contact between a rigid rough surface and a layered half-space consisted of the coating with thickness $\delta 1$ and the substrate. By using the stiffness model of a layered half-space, the elastic characteristic are determined for every contacting asperity. The system of transcendental equations which allow to determine the dependence of the density of gaps in case of contact through coating layer on the roughness parameters, material properties, coating thickness and applied load is given. The characteristic curves showing the dependence of the density of gaps in the joint for different coating thickness are given.
\end{abstract}

Keywords- rough surface, spherical asperity, elastic contact of asperities, volume of gaps, density of joint, thin-layer coatings, layered elastic half-space.

\section{INTRODUCTION}

A promising way to increase seals and friction joints ${ }^{6}$ operational factors is the surfacing polymeric covering on their work surfaces anti-frictional films. Operation experiment has shown that the sealing ability antifriction properties are determined not only by the properties, but also the thickness of the coating material [1]. Long experience of the authors in improving the methods of calculation of tightness of joints show that the density of gaps and in the seal junction and the relative contact area are the critical characteristics. The influence of coating thickness on the relative area for different types of contact is considered in $[2,3]$. At the same time, there are no data of the influence of coating thickness on the density of gaps in the joint. It has defined the purpose of the present paper.

\section{CONTACT BETWEEN A ROUGH SURFACE AND A HOMOGENEOUS ELASTIC HALF-SPACE}

We use the discrete model of a rough surface, in which microasperities are presented by identical spherical segments with the distribution of segments' peaks on height corresponds to the bearing profile curve of the real surface
[4]. To describe the distribution of the reference curve is used incomplete beta function:

$$
\eta(\varepsilon)=\frac{B_{\varepsilon}(\alpha, \beta)}{B(\alpha, \beta)}
$$

Where $\mathrm{B}_{\varepsilon}(\alpha, \beta)$ and $\mathrm{B}(\alpha, \beta)$ are incomplete and complete beta functions;

$\alpha=\left(\frac{R_{p}}{R_{q}}\right)^{2}\left(\frac{R_{\max }-R_{p}}{R_{\max }}\right)-\frac{R_{p}}{R_{\max }}, \beta=\alpha\left(\frac{R_{\max }}{R_{p}}-1\right) ;$

Where $R_{p}, R_{q}, R_{\max }$ are height roughness parameters according to ISO 4281/1-1997.

In this case, the density of the asperities distribution on height function is

$$
\varphi_{n}^{\prime}(u)=\frac{u^{\alpha-2}(1-u)^{\beta-2}[(\alpha-1)(1-u)-(\beta-1) u]}{\varepsilon_{s}^{\alpha-1}\left(1-\varepsilon_{s}\right)^{\beta-1}},
$$

Where $\varepsilon_{s}$ is determined from the condition $\varphi_{n}\left(\varepsilon_{s}\right)=1$, $\omega R_{\max }$ is the height of a spherical segment, $\omega=1-\varepsilon_{s}$.

The radius of the spherical segment is

$$
r=\frac{a_{c}^{2}}{2 \omega R_{\max }}
$$

Where $a_{c}$ is the radius of the base of the spherical segment.

The problem of the density of gaps upon contact between a rigid rough surface and a half-space was considered in [5]. As it follows from [5], to find the volume of intercontact space it is necessary to determine the volume of gaps attributable to the single contacting or noncontacting asperities as

$$
V_{c}=\left\{\begin{array}{l}
V_{r i}=2 \pi \int_{a_{r i}}^{a_{c}}\left[z_{10}(\rho)-z_{20}(\rho)\right] \rho d \rho, \\
V_{o i}=2 \pi \int_{0}^{a_{c i}}\left[z_{1 r}(\rho)-z_{2 r}(\rho)\right] \rho d \rho,
\end{array}\right.
$$


Where $z_{10}$ and $z_{20}$ are the equations describing the surfaces of noncontacting asperities and a half-space; $z_{1 r}$ and $z_{2 r}$ are the equations describing the surfaces of contacting asperities and a half-space.

Then the total volume of intercontact space in the joint can be written as

$$
V_{c}=\sum_{i=1}^{n_{r}} V_{r i}+\sum_{i=1}^{n_{c}-n_{r}} V_{o i}
$$

And the corresponding density of gaps can be written as

$$
\begin{aligned}
& \Lambda(\varepsilon)=\frac{V_{c}}{A_{c} R_{\max }}= \\
& =\frac{1}{A_{c i} R_{\max }}\left[\int_{0}^{\min \left(\varepsilon, \varepsilon_{s}\right)} V_{r i} \varphi_{n}^{\prime}(u) d u+\int_{\min \left(\varepsilon, \varepsilon_{s}\right)}^{\varepsilon_{s}} V_{o i} \varphi_{n}^{\prime}(u) d u\right] .
\end{aligned}
$$

Given that $V_{r i}=A_{c i} R_{\max } \Lambda_{r i}$ and $V_{o i}=A_{c i} R_{\max } \Lambda_{o i}$, Eq. (6) can also be written as

$$
\Lambda(\varepsilon)=\int_{0}^{\min \left(\varepsilon, \varepsilon_{s}\right)} \Lambda_{r i} \varphi_{n}^{\prime}(u) d u+\int_{\min \left(\varepsilon, \varepsilon_{s}\right)}^{\varepsilon_{s}} \Lambda_{o i} \varphi_{n}^{\prime}(u) d u
$$

Let us define the equations describing the surface asperities and a half-space included in Eq. (7).

Fig. 1 shows the schematic illustration of a contact of a single asperity. The index 1 refers to an asperity, the index 2 refers to a half-space.

Initially the equation of $i$-th asperity's section can be written as

$$
z_{10}=u R_{\max }+\frac{\rho^{2}}{2 r}=u R_{\max }+\frac{\omega R_{\max } \rho^{2}}{a_{c}^{2}},
$$

The equation of a half-space is $z_{20}=0$, it is held also for a noncontacting asperity.

If the displacement of an asperity is equal $\varepsilon<u$, then contact between it and a half-space does not occur. Then, for a noncontacting asperity we can write

$$
z_{10}=\frac{\omega R_{\max } \rho^{2}}{a_{c}^{2}}+(u-\varepsilon) R_{\max } \text { or } z_{10}=\omega R_{\max }\left(x^{2}+\frac{u-\varepsilon}{\omega}\right)(9)
$$

If the displacement of an asperity is equal $\varepsilon>u$, then contact between it and a half-space occurs. Then, for a contacting asperity we can write

$$
z_{1 r}=\frac{\omega R_{\max } \rho^{2}}{a_{c}^{2}}-(\varepsilon-u) R_{\max } \text { or } z_{1 r}=\omega R_{\max }\left(x^{2}-\frac{\varepsilon-u}{\omega}\right)(10)
$$

Where $x=\rho / a_{c}$.

The pressure distribution over the contact area is Hertzian, that is

$$
p(\rho)=p_{0}\left(1-\frac{\rho^{2}}{a_{r}^{2}}\right)^{\frac{1}{2}}=\frac{3}{2} p_{m}\left(1-\frac{\rho^{2}}{a_{r}^{2}}\right)^{\frac{1}{2}},
$$

Where $p_{0}$ and $p_{m}$ are the maximum pressure and the average contact area.

According to [12], the displacement of a half-space depending on the load of the form (11) is

$$
\begin{aligned}
& z_{2 r}=u_{z}=-\frac{3 p_{0}}{2 E^{*}} \cdot \frac{a_{r}^{2}}{\rho} \cdot{ }_{2} F_{1}\left(\frac{1}{2}, \frac{1}{2} ; \frac{5}{2} ; \frac{a_{r}^{2}}{\rho^{2}}\right)= \\
& =-\frac{p_{m}}{E^{*}} \cdot \frac{a_{r}^{2}}{\rho} \cdot{ }_{2} F_{1}\left(\frac{1}{2}, \frac{1}{2} ; \frac{5}{2} ; \frac{a_{r}^{2}}{\rho^{2}}\right) .
\end{aligned}
$$

Where ${ }_{2} F_{1}(a, b ; c ; z)$ is the Gaussian hypergeometric function.

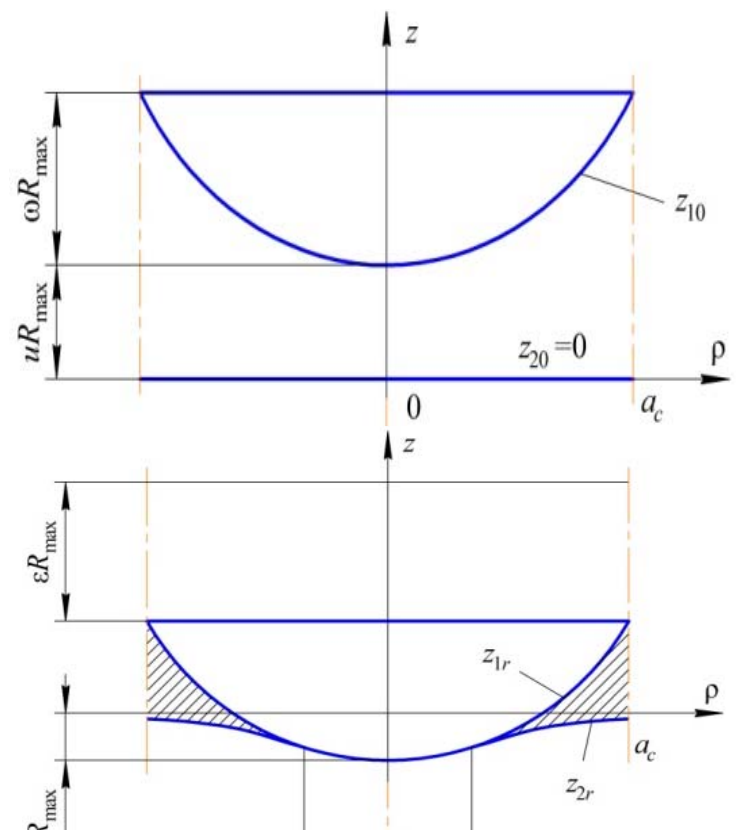

Figure 1. Schematic illustration of single spherical asperity's contact: a) the original location; b) the displacement of an asperity equal to $\omega R_{\max }$.

Given that $a_{r}^{2} / a_{c}^{2}=\eta_{i}$, we have

$$
z_{2 r}=-\frac{p_{m i} a_{c}}{E^{*}} \cdot \frac{\eta_{i}}{x} \cdot{ }_{2} F_{1}\left(\frac{1}{2}, \frac{1}{2} ; \frac{5}{2} ; \frac{\eta_{i}}{x^{2}}\right)
$$


From Hertzian formulae with taking into account Eq. (4), we obtain

$$
\frac{p_{m i}}{E^{*}}=\frac{8 \eta_{i}^{0,5}}{3 \pi} \cdot \frac{\omega R_{\max }}{a_{c}} .
$$

Then we have

$$
z_{2 r}=-\frac{8 \eta_{i}^{1,5}}{3 \pi} \cdot \frac{\omega R_{\max }}{x}{ }_{2} F_{1}\left(\frac{1}{2}, \frac{1}{2} ; \frac{5}{2} ; \frac{\eta_{i}}{x^{2}}\right) .
$$

From [2] for elastic contact, we have

$$
\eta_{i}=\eta_{i}(\varepsilon, u)=\frac{\varepsilon-u}{2 \omega}
$$

Integrating Eq. (5) with taking into account sections of contacting and noncontacting asperities and a half-space, we have

$$
\begin{gathered}
\frac{V_{0 i}}{\pi a_{c}^{2} R_{\max }}=\Lambda_{0 i}=\omega\left(\frac{1}{2}-\frac{\varepsilon-u}{\omega}\right), \\
\frac{V_{r i}}{\pi a_{c}^{2} R_{\max }}=\Lambda_{r i}= \\
=\omega\left(\begin{array}{l}
\frac{1}{2}-\frac{\varepsilon-u}{\omega}\left(1-\eta_{i}\right)- \\
-2 \eta_{i}^{2}+\frac{16 \eta_{i}^{1.5}}{3 \pi}{ }_{2} F_{1}\left(-\frac{1}{2}, \frac{1}{2} ; \frac{5}{2} ; \eta_{i}\right)
\end{array}\right) .
\end{gathered}
$$

Substituting the above Eqs. (17) and (18) into Eq. (7), we can determine the density of gaps in the joint: $\Lambda(\varepsilon)$ depending on the relative magnitude $\varepsilon$.

For given model of roughness, the mean contact pressure applied to the joint [4] is

$$
q_{c}(\varepsilon)=\int_{0}^{\min \left(\varepsilon, \varepsilon_{s}\right)} q_{c i}(\varepsilon) \varphi_{n}^{\prime}(u) d u .
$$

Given that $q_{c i}=q_{m i} \eta_{i}$, we have

$$
\frac{q_{c} a_{c}}{E^{*} \omega R_{\max }}=F_{q}=\frac{8}{3 \pi} \int_{0}^{\min \left(\varepsilon, \varepsilon_{s}\right)} \eta_{i}(\varepsilon, u) \varphi_{n}^{\prime}(u) d u .
$$

\section{THE ELASTIC CHARACTERISTIC OF A LAYERED HALF- SPACE}

Let us consider a layered elastic half-space consisting of coating with thickness $\delta_{1}$, the elastic characteristics $v_{1}$ and $E_{1}$ and the substrate with the elastic characteristics $v_{0}$ and $E_{0}$. Using the stiffness model of a layered half-space, the authors $[3,7]$ determined its reduced elastic characteristic depending on the coating thickness and elastic constants of the substrate and the coating. For the reduced modulus of elasticity, it is obtained that

$$
E_{01}^{*}=E_{1}^{*} \cdot F_{1}
$$

$$
F_{1}=K_{1}(0)\left[\frac{\left(K_{1}(0)-K_{1}(\bar{\delta})\right)^{2}}{K_{01}(0)-K_{01}(\bar{\delta})}+K_{1}(\bar{\delta}) \frac{K_{0}(\bar{\delta})}{K_{01}(\bar{\delta})} \cdot \frac{E_{1}^{*}}{E_{0}^{*}}\right]^{-1}
$$

Where $\bar{\delta}=\delta / a$;

$$
K_{i}(\bar{z})=\operatorname{arccot}(\bar{z})+\frac{v}{1-v} \bar{z}(1-\bar{z} \operatorname{arccot}(\bar{z})) .
$$

To determine $K_{01}\left(\bar{\delta}_{1}\right)$ it is need to know $v_{01}$. Since the values of the function $K_{i}(\bar{z})$ for $v=0.25 \ldots 0.5$ vary slightly, then for the first iteration we should take $v_{01}=0.5\left(v_{0}+v_{1}\right)$. For subsequent iterations with an error of less than $1 \%$ can be taken

$$
v_{01}=v_{1}+\left(v_{0}-v_{1}\right) \frac{1-F_{1}^{-1}}{1-E_{1}^{*} / E_{0}^{*}} .
$$

The number of iterations depends on the assumed accuracy. In the case of contact between a smooth rigid sphere and a layered half-space convergence of bodies, the contact radius and the maximum pressure are defined by equations

$$
w_{01}=w_{1} \cdot F_{1}^{-\frac{2}{3}}, a_{01}=a_{1} F_{1}^{-\frac{1}{3}}, p_{01}=p_{0} F_{1}^{\frac{2}{3}} \text {. }
$$

\section{MODELING OF CONTACT OF A RIGID ROUGH SURFACE THROUGH AN ELASTIC COATING LAYER}

In case of contact between a rough surface with an elastic layered half-space, $\bar{\delta}_{1 i}$ can be defined by equation

$$
\bar{\delta}_{i}=\frac{\delta}{a_{r}}=\frac{\delta}{a_{c}} \frac{a_{c}}{a_{r}}=\gamma \eta_{i}^{-0.5},
$$


Where $\gamma=\delta_{1} / a_{c}$ is the relative thickness of the coating; $\eta_{i}=a_{r}^{2} / a_{c}^{2}$ is the relative contact area for a single asperity; $a_{c}$ is the radius of the area attributable by one asperity.

Thus, for every contacting asperity according to the Eq. (21), we have

$$
E_{01}^{*}\left(\gamma, \eta_{i}\right)=E_{1}^{*} F_{1}\left(\gamma, \eta_{i}\right)
$$

Where $F_{1}\left(\gamma, \eta_{i}\right)$ is defined by the Eq. (22) with taking into account Eq. (23).

In case of contact between a rigid rough surface with a layered elastic half-space it should be taking into account that according to the Eqs. (24) and (26) we have

$$
\begin{gathered}
\eta_{i}(\varepsilon, u)=\eta_{1 i}(\varepsilon, u) \cdot F_{1 i}^{-\frac{2}{3}}(\gamma, \varepsilon, u), \\
E_{01}^{*}(\gamma, \varepsilon, u)=E_{1}^{*} \cdot F_{1 i}(\gamma, \varepsilon, u),
\end{gathered}
$$

Then, as in the Eqs. (18) and (20) we obtain

$$
\begin{aligned}
& \Lambda_{r i}(\gamma, \varepsilon)= \\
& \left.=\omega\left(\begin{array}{l}
\frac{1}{2}-2 F_{1 i}^{-\frac{2}{3}}(\gamma, \varepsilon, u) \eta_{i}(\gamma, \varepsilon)+ \\
+\frac{16 F_{1 i}^{-1}(\gamma, \varepsilon, u) \eta_{i}^{1.5}(\gamma, \varepsilon)}{3 \pi} \times \\
\times{ }_{2} F_{1}\left(-\frac{1}{2}, \frac{1}{2} ; \frac{5}{2} ; F_{1 i}^{-\frac{2}{3}}(\gamma, \varepsilon, u) \eta_{i}(\gamma, \varepsilon)\right.
\end{array}\right)\right) ; \\
& F_{q 1}(\gamma, \varepsilon)=\frac{q_{c} a_{c}}{E_{1}^{*} \omega R_{\max }}=\frac{8}{3 \pi} \times \\
& \times \int_{0}^{\min \left(\varepsilon, \varepsilon_{s}\right)} F_{1 i}(\gamma, \varepsilon, u) F_{1 i}^{-1}(\gamma, \varepsilon, u) \eta_{i}^{1.5}(\varepsilon, u) \varphi_{n}^{\prime}(u) d u .
\end{aligned}
$$

The equation for $V_{o i}(\gamma, \varepsilon)$ will be similar to the Eq. (17). The density of gaps in case of contact between a rigid rough surface and a layered elastic half-space is defined by equation

$$
\begin{aligned}
& \Lambda_{c}(\gamma, \varepsilon)=\int_{0}^{\min \left(\varepsilon, \varepsilon_{s}\right)} \Lambda_{r i}(\gamma, \varepsilon) \varphi_{n}^{\prime}(u) d u+ \\
& +\int_{\min \left(\varepsilon, \varepsilon_{s}\right)}^{\varepsilon_{s}} \Lambda_{o i}(\gamma, \varepsilon) \varphi_{n}^{\prime}(u) d u .
\end{aligned}
$$

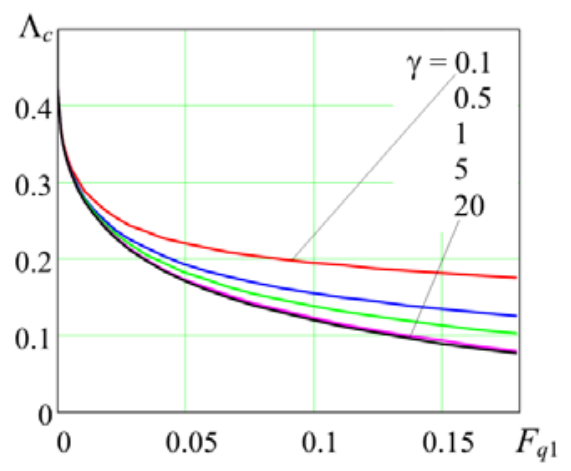

Figure 2. The relations $\Lambda_{c}\left(F_{q 1}\right)$ for different values of the relative thickness of the coating, for $E_{0}=200 \mathrm{GPa}, v_{0}=0.3 ; E_{0}=20 \mathrm{GPa}, v_{0}=0.3$

The relations $\Lambda_{c}\left(F_{q 1}\right)$ for different values of the relative thickness of the coating computed using the Eqs. (17), (29) (31) are shown in Fig. 2.

\section{CONCLUSIONS}

1. Using the discrete roughness model, the equations for determining the density of gaps in the joint are obtained with taking into account the displacements of a half-space by every contacting single asperity.

2. The method for determining the density of gaps by contacting between a rigid rough surface and a homogeneous material was applied to the contact with a layered elastic half-space is given. In this case we used the stiffness model of layered body to determine the reduced modulus of elasticity for any asperities.

3 . The influence of the relative coating thickness on the density of gaps in the joint is shown.

\section{REFERENCES}

[1] A.P. Makushkin "Polymers in friction and seals at low temperatures," Moscow: Mashinostroenie, 1993, 228 p. (in Russian).

[2] P.M.Ogar, O.V. Maksimova, V.A.Tarasov "The influence of the coating thickness on the relative area of contact conjugations of machine parts," Systems. Methods. Technologies, No 2, 2009, pp. 13-15. (in Russian).

[3] P.M. Ogar, V.A. Tarasov, D.B. Gorokhov "The influence of coating thickness on the relative area of tribounits contact," Advanced Materials Research, vol. 1061-1062, 2015, pp. 614-618.

[4] V.A. Tarasov, D.B. Gorokhov, A.S. Kozhevnikov "Modeling of contact of a rigid rough surface through the coating layer," Trudy Bratskogo gosudarstvennogo universiteta. Seriya: Estestvennye I inzhenernye nauki, vol. 2, 2014, pp. 47-55. (in Russian).

[5] P.M. Ogar, V.A. Tarasov, A.V. Turchenko "Rough asperity and elastic-plastic half-space contact," Systems. Methods. Technologies, No 1, 2012, pp. 17-22. (in Russian).

[6] P.M. Ogar, V.A. Tarasov "Influence of axisymmetric form of load on stressedly-deformed state of elastoplastic half-space," Systems. Methods. Technologies., No 5, 2010, pp. 14-20. (in Russian).

[7] P.M. Ogar, V.A. Tarasov "Determination of the Elastic Characteristics of Bodies with Thin Coatings," Advanced Materials Research, vol. 677, 2013, pp. 267-272. 